\title{
MODELING THE EFFECT OF SUBTASK BOUNDARIES ON DRIVER GLANCE BEHAVIOR
}

\author{
Ja Young Lee and John D. Lee \\ University of Wisconsin-Madison \\ Madison, WI, USA \\ Email: jayoung.lee@wisc.edu,john.d.lee@wisc.edu
}

\begin{abstract}
Summary: Studies of multitasking while driving have shown that drivers tend to switch attention at subtask boundaries. It is also known that the uncertainty of roadway information plays a significant role in attention switching. Yet, these two approaches have not been modeled together. In this study, we create an attention switching model that accounts for both subtask boundaries and uncertainty, and use Approximate Bayesian Computation-Markov Chain Monte Carlo (ABCMCMC) to determine the weight between the two factors, based on the empirical data. The weight was calculated for each of two different types of tasks, text reading and entry, that have subtask boundaries with different characteristics. We found that the subtask boundary in the text reading task nudged drivers to discontinue the distracting task and switch attention back to the road more than the subtask boundary in the text entry task. This study suggests that task structure may play a role in generating long glances.
\end{abstract}

\section{INTRODUCTION}

In-vehicle infotainment systems draw drivers' attention away from the road. As the duration of such distraction gets longer, drivers are more likely to miss critical events on the road, such as lead vehicle braking, red lights, stop signs, and so forth, and this could lead to crashes. As Horrey and Wickens (2007) pointed out, crashes are induced by the exceptional behavior rather than the norm, in particular, $85^{\text {th }}$ percentile glance duration rather than mean glance duration. Hence, it is important to pay more attention to the microstructure of interactions that lead to long glances to reduce the risk of crashes. Below we review factors that affect drivers' attention switching, which helps to determine the duration of glances away from the road.

Drivers demonstrate strategic and dynamic behaviors to meet environmental demands and task characteristics. A model by Senders et al. (1967) accounted for the environmental demands: drivers switch attention when the uncertainty about the road and vehicle state exceeds a threshold, to reduce the discomfort of high uncertainty. Other studies have proposed that task characteristics influence the switching behavior (e.g., Janssen, Brumby, \& Garnett, 2012): a task can be decomposed into multiple subtasks, and drivers tend to switch at the boundaries of subtasks rather than in the middle of the subtask, because switching at subtask boundary benefits people by reducing workload (Salvucci \& Bogunovich, 2010).

In this study, we create a model that simulates attention switching behavior by linearly combining the effects of both environmental demands (i.e., uncertainty accumulation) and task characteristics (i.e., subtask boundary), and compared the model results with the experiment results where participants performed secondary tasks while driving. This model goes beyond a 
simple driver model, for example, a model with an internal time limit after which the driver switches attention from the distracting task to the driving task (e.g., Kujala \& Salvucci, 2015). We assume that the attention switching is a weighted combination of two probabilities: (1) the probability of switching is 1 at subtask boundaries, 0 otherwise and (2) probability of switching attention that increases with uncertainty and reaches 1 when crossing threshold. For example, if weight of the former (boundary) is 1 and the weight of the latter (uncertainty) is 0 , the driver switches attention only at the subtask boundary. On the other hand, if the weight of boundary is 0 and the weight of uncertainty is 1 , the driver only switches when uncertainty exceeds threshold and does not respond to the subtask boundary.

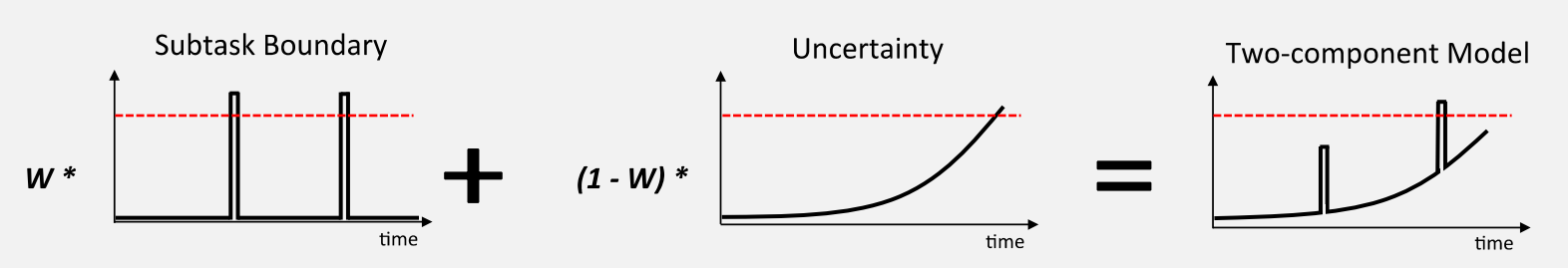

Figure 1. Two-component model. Subtask boundary and uncertainty is weighted by $w$ and $1-w$, respectively

To find the weights of the subtask boundary and uncertainty, we use ABC-MCMC (Approximate Bayesian Computation - Markov Chain Monte Carlo), which makes it possible to estimate parameters of complex, dynamic, and non-linear models (Hartig, Calabrese, Reineking, Wiegand, \& Huth, 2011). The ABC method first runs the model with a parameter sampled from a prior distribution. The outcome of the model is then compared to the data observed from the system being modeled. If the two are similar, the parameter is accepted and accumulated into the posterior distribution of the parameter. The model is again simulated with another parameter that is close to the parameter just used, as defined by the MCMC sampling method (see Turner \& Van Zandt, 2012). If the simulated outcome is not similar to the observed data, a random parameter is sampled from the prior distribution. This process is repeated until we obtain a posterior distribution that approximates the true distribution of the parameter values. To compare the similarity between the observed data and simulated data, ABC uses 'summary statistics' of the data (e.g., mean, quartiles, etc.). Summary statistics characterize the essential elements of the observed behavior, and is central to the quality of the approximation (Turner \& Van Zandt, 2012).

The weight parameter is estimated for two tasks, text reading and text entry. In the experiment, participants (1) read sentences displayed on two screens, which required drivers to press a button to transition between two screens, and (2) type a compound word (e.g., streetlights) using a touchscreen interface, which implicitly requires memory retrieval of a subword. We use ABCMCMC to estimate the parameter that represents the extent that the subtask boundaries (i.e., button press and memory retrieval) account for switching visual attention between the device and the roadway. 


\section{METHODS}

\section{Experiment}

Participants. Forty-eight participants from four age groups (18-24, 25-39, 40-54, and 55+ years old) with an equal number of participants across groups and equal number of males and females within each group completed the study. All participants performed two distracting tasks (i.e., reading and entry) in a counterbalanced order while driving a vehicle simulator.

Driving Task and Apparatus. NADS MiniSim ${ }^{\mathrm{TM}}$ driving simulator with 65-inch screen was used for the experiment. The participants were instructed to drive in the right lane of an undivided, four-lane straight highway at a speed of $50 \mathrm{mph}(80 \mathrm{~km} / \mathrm{h})$ and maintain a fixed following distance of 220 feet $(67 \mathrm{~m})$ with the lead vehicle for the entire experiment. A GoPro camera was mounted to record drivers' head and eye movements.

Secondary Task and Apparatus. Each participant completed a three-minute practice drive and received feedback on their performance maintaining a 220 -foot $(67 \mathrm{~m})$ following distance. Each participant completed 6 trials of text reading task and 6 trials of text entry task on a 7 -inch touchscreen attached at a center-stack position. For text reading trials, the participants read the first page and pressed 'next' to read the second page of the screen. They pressed 'enter' when finished reading the second page. Each sentence was about 130 characters long. We assumed that hand movement to press 'next' acts as a natural subtask boundary for the reading task. For text entry trials, the participants heard word audio prompts and used the keyboard at the bottom of the screen to enter the word they heard. They pressed 'enter' when done. Each of the six words was a compound word that consisted of 12 letters. We assumed that the end of the first subword acts as a subtask boundary, signaled by cognitive memory cue, as people retrieve two chunks of words from their memory to form a compound word. Each condition began with four practice trials. The trials within a task were randomized.

Data Preparation. Eye glance data was manually coded following the NHTSA visual-manual guidelines (2014) using a video logging tool Morae ${ }^{\circledR}$. A glance is defined as the time from the moment the direction of gaze moves toward the touchscreen to the moment it moves away from it, as defined in SAE standard (SAE J2396).

\section{Driver Attention Switching Model}

We created a driver model that performs two types of tasks, text reading and text entry, while driving. The virtual drivers in the model switched attention from the distracting task to the roadway in a probabilistic way, as a function of a linear combination of subtask boundary and amount of uncertainty. While looking away, uncertainty was accumulated following the formula (2) (Senders et al. 1967). For every switch of visual attention from the road to the task screen, 200ms switch cost was added. If the switch was not at the subtask boundary, additional switch cost of 100ms was added (Janssen, Brumby, Garnett 2012). We assumed that each key press takes 480ms. Reading and understanding one word was 200ms (Anderson et al., 2004). On-road glance duration was sampled from a lognormal distribution $\sim L N\left(-0.260,0.778^{2}\right)$, according to the result of the current experiment $(M=1.09 \mathrm{~s}, S D=1.24 \mathrm{~s})$. 


\section{ABC-MCMC Parameter Fitting}

The weight of the subtask boundary in relation to uncertainty, $\alpha$ in formula (1), was estimated using ABC-MCMC. The higher $\alpha$ value indicates greater influence of the task boundary on attention switching. Note that the parameter $\alpha$ was estimated two times, one for text reading task and the other for text entry.

Prob(visual attention switch $)=\alpha *$ task boundary $+(1-\alpha) *$ uncertainty

The task boundary term was either 0 or 1 at any moment. In the text reading task, it was 1 only when the next action is to press 'next' to read the second page. In the text entry task, it was 1 only when the first subword was completely entered and the next action was to enter the first letter of the second subword. For the uncertainty term, accumulated uncertainty was divided with the threshold $U$. Thus, the uncertainty accumulates from 0 to 1 as time away from the road increases, as shown in (2). The first term of numerator represents lost information over time $(H$ : road information density, $D$ : weight on information, $F$ : rate of forgetting) and the second term represents vehicle instability $(K$ : constant related to vehicle stability relative to road, $V$ : vehicle velocity, $t$ : time occluded). The constants were: $H=15.6, D=0.3, F=6.1, K=.0002$, and $U=$ 4.9. These values are the averages of values reported in Senders et al. (1967).

uncertainty $=\left(H * D *\left(1-e^{-\left(\frac{V}{D}+\frac{1}{F}\right)}\right) * t+K * V^{2} * t^{1.5}\right) / U$

As a prior distribution of $\alpha$, we used a uniform distribution between 0 and 1 , which reflects our lack of information regarding the prior distribution. Five summary statistics were used to compare the glance pattern of the simulation and the experiment: the number of glances, mean glance duration, total glance duration, maximum duration, and percentage of long glances over two seconds, per trial. The acceptance criterion for each of summary statistic was one standard deviation from the true mean. For each task type 10,000 simulation runs were performed.

\section{RESULTS}

Each of the 48 drivers completed 6 reading and 6 entry tasks. For each trial, five glance features were calculated, and the features were summarized over all trials as listed in Table 1 . The summarized glance features were used as summary statistics for the ABC-MCMC.

Table 1. Off-the-road glance features observed from the experiment

\begin{tabular}{ccc}
\hline & Text Reading Task & Text Entry Task \\
& Mean (SD) & Mean (SD) \\
\hline Number of Glances & $6.56(3.02)$ & $6.59(2.95)$ \\
Mean Glance Duration (sec) & $1.63(0.41)$ & $2.01(0.78)$ \\
Total Glance Duration (sec) & $10.25(4.51)$ & $12.00(4.65)$ \\
Maximum Glance Duration (sec) & $2.60(0.82)$ & $3.25(1.21)$ \\
\% of Long Glances $(>\mathbf{2} \mathbf{~ s e c )}$ & $9.62(9.33)$ & $15.56(11.66)$ \\
\hline
\end{tabular}

Overall, the subtask boundary effect accounted for $75-85 \%$ of the attention switching. The subtask boundary in the reading task was more pronounced $(M=.85, S D=.04)$ than the subtask 
boundary in the entry task $(M=.75, S D=.06)$. Recalling that $\alpha$ indicates relative influence of task boundary in attention switching, higher $\alpha$ in reading task implies that the drivers were influenced by the task boundary in the reading task, which was signaled by motor movement, more than the boundary in the entry task signaled by the retrieval of a memory chunk.

Table 2. Summary of posterior distribution of $\alpha$ for the read and entry tasks.

\begin{tabular}{ccccccc}
\hline & N & Mean & SD & Median & Min & Max \\
\hline Reading & 772 & .85 & .04 & .85 & .72 & .96 \\
Entry & 1,146 & .75 & .06 & .75 & .54 & .90 \\
\hline
\end{tabular}

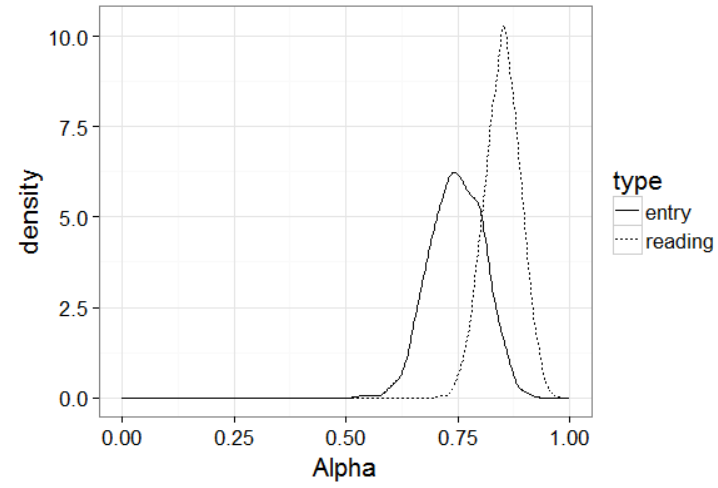

Figure 2. Posterior distribution of $\alpha$ for the read tasks (solid) and the entry tasks (dashed)

With the approximated $\alpha$ distribution, we simulated glance behavior of 48 virtual drivers. For each of reading and entry task, $48 \alpha$ values were sampled from the posterior distribution and the model was run for each value. Results showed that the simulated glance duration distribution was similar to the glance distribution of the experiment (Figure 3).

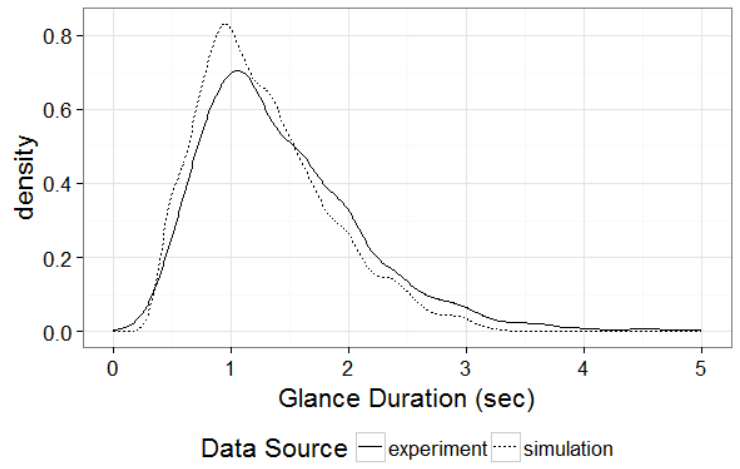

(a) Reading task

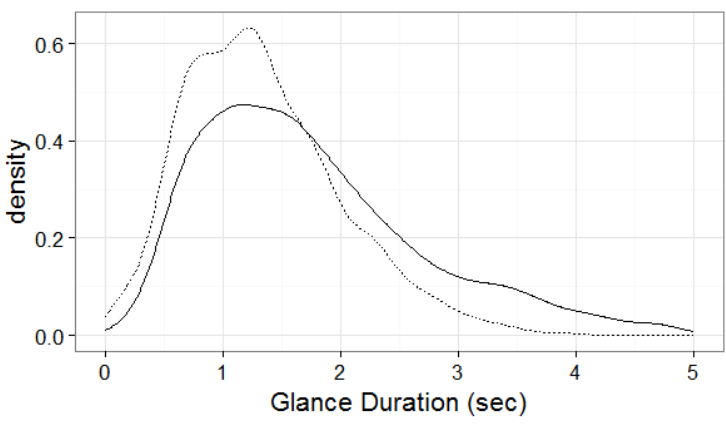

Data Source - experiment …. simulation

(b) Entry task

Figure 3. The simulated glance duration (dashed line) resembled the glance duration from the experiment (solid line).

\section{DISCUSSION}

Glance duration is dynamically influenced by task structure and uncertainty about the road. In the current study, we modeled when drivers switch attention by considering both internally accumulated uncertainty and subtask boundaries. The ABC-MCMC technique enabled us to estimate the weight on the effect of subtask boundaries, and the resulting behavior of the model closely resembles actual driver behavior. Considering the dynamics of uncertainty and subtask boundaries has not been systematically studied in previous literature. For instance, Senders (1967) estimated the duration that drivers can drive with eyes occluded, based on how fast 
roadway information is and accrued and forgotten. This approach cannot describe strategies that drivers adapt to minimize cognitive effort in performing the distracting task, such as switching attention at subtask boundaries rather than in the middle of a subtask. Although other researchers studied the effects of such subtask boundaries, it was unclear in these studies where else in the task the drivers switched attention. By combining two approaches, we can estimate how the task structure influences the duration of glances away from the road.

In the reading task, the boundary was explicitly signaled by the movement of a hand, where the driver had to press 'next' to display the next half of the message, reflecting a change in the modality used for task, from visual to manual. On the other hand, in the entry task, the boundary was implicitly signaled by the retrieval of a memory chunk. Our results complies with a study of Iqbal \& Bailey (2006), where people are more likely to interleave tasks based on the subtask boundary proportional to the degree that it reduces resumption time. The results also complement the work of Janssen, Brumby, \& Garnett (2012) who found drivers switched at natural break points signaled by motor cues (repositioning of fingers) to a greater degree than cognitive cues (structure of phone number in memory).

Driver models have been mostly deterministic and fitted with least squares method. However, human behavior is not deterministic. $\mathrm{ABC}-\mathrm{MCMC}$ is a method that can estimate parameters of a stochastic model that closely mimics the variable behavior of driver. $\mathrm{ABC}-\mathrm{MCMC}$ is a new, useful method made possible by the power of modern computers. Essentially, it runs a model with numerous potential parameters but limits the sampling space based on the result of the preceding run of the model, increasing the efficiency. Even so thousands of model runs are required. From our observation, this method reliably generated the posterior distribution of $\alpha$ over multiple tests. In addition, when we used these parameters in the model, the model produced similar behaviors as people. Because we used the proportion of long glances and maximum glance duration as summary statistics, the model was tuned to match the potentially dangerous long glances that Horrey \& Wickens (2007) emphasized.

In the future, we hope to extend the model and test whether a greater number of subtask boundaries benefits driving safety. We can also include more parameters that account for other aspects of behavior, such as individual differences in the tolerance of uncertainty or response to task boundary (Lee, Gibson, \& Lee, 2015), to enhance external validity of the model to real world driving. The model developed in this study also has implications for future models that extend beyond driver. If a driver is looking away from the road during a safety-critical event, such as lead vehicle braking, the glance duration at the point determines how long until the driver looks back to the road. This in turn can help determine whether the event is a crash or near-crash, or high speed collision with high injury risk or low speed collision with low injury risk. Thus, the newly proposed model, which generates sequence of glances that have a distribution of durations that matches actual drivers, may be used to estimate the crash risk associated with various system designs. 


\section{ACKNOWLEDGEMENT}

Data used in this paper is derived from research supported by the U.S. Dept. of Transportation National Highway Traffic Safety Administration under Contract No. DTNH22-11-D-00237. Any opinions, findings, and conclusions or recommendations expressed in this publication are those of the authors and do not necessarily reflect the views of the NHTSA or the U.S. DOT.

\section{REFERENCES}

Anderson, J. R., Bothell, D., Byrne, M. D., Douglass, S., Lebiere, C., \& Qin, Y. (2004). An Integrated Theory of the Mind. Psychological Review, 111, 1036-1060.

Hartig, F., Calabrese, J. M., Reineking, B., Wiegand, T., \& Huth, A. (2011). Statistical inference for stochastic simulation models - theory and application. Ecology Letters, 14, 816-827.

Horrey, W., \& Wickens, C. (2007). In-vehicle glance duration: Distributions, tails, and model of crash risk. Transportation Research Record: Journal of the Transportation Research Board, 2018, 22-28.

Janssen, C. P., Brumby, D. P., \& Garnett, R. (2012). Natural break points: The influence of priorities and cognitive and motor cues on dual-task interleaving. Journal of Cognitive Engineering and Decision Making, 6, 5-29.

Kujala, T., \& Salvucci, D. D. (2015). Modeling visual sampling on in-car displays: The challenge of predicting safety-critical lapses of control. International Journal of HumanComputer Studies, 79, 66-78.

Lee, J. Y., Gibson, M., \& Lee, J. D. (2015). Secondary task boundaries influence drivers' glance durations. In Proceedings of the 7th International Conference on Automotive User Interfaces and Interactive Vehicular Applications - AutomotiveUI '15 (pp. 273-280). New York, New York, USA: ACM Press.

SAE J2396 Definitions and Experimental Measures Related to the Specifications of Driver Visual Behavior Using Video Based Techniques.

Salvucci, D. D., \& Bogunovich, P. (2010). Multitasking and monotasking: The effects of mental workload on deferred task interruptions. In Proceedings of the SIGCHI conference on human factors in computing systems (pp. 85-88).

Senders, J. W., Kristofferson, A. B., Levison, W. H., Dietrich, C. W., \& McDonald, J. W. (1967). The attentional demand of automobile driving. Highway Research Record.

Turner, B. M., \& Van Zandt, T. (2012). A tutorial on approximate Bayesian computation. Journal of Mathematical Psychology, 56, 69-85. 